\title{
Fixed-Time Synchronization of the New Single-Parameter Chaotic System
}

\author{
Yifeng Wei, ${ }^{1}$ Xia Qing $\mathbb{D}^{2},{ }^{2}$ Xie Chengrong $\mathbb{D}^{3},{ }^{3}$ and Yuhua Xu $\mathbb{D}^{4}$ \\ ${ }^{1}$ School of Economy and Management, Hanjiang Normal University, Shiyan, Hubei 442000, China \\ ${ }^{2}$ School of Economics, Wuhan Donghu University, Wuhan 430212, China \\ ${ }^{3}$ School of Statistics and Mathematics, Nanjing Audit University, Nanjing, Jiangsu 211815, China \\ ${ }^{4}$ School of Finance, Nanjing Audit University, Nanjing, Jiangsu 211815, China \\ Correspondence should be addressed to Xia Qing; 77218434@qq.com
}

Received 11 June 2020; Accepted 20 July 2020; Published 3 August 2020

Academic Editor: Akif Akgul

Copyright $\odot 2020$ Yifeng Wei et al. This is an open access article distributed under the Creative Commons Attribution License, which permits unrestricted use, distribution, and reproduction in any medium, provided the original work is properly cited.

\begin{abstract}
This paper advances a new single-parameter chaotic system with a simple structure, and some basic dynamic behavior of the new single-parameter system is discussed, such as equilibria, dissipativity, the existence of an attractor, Lyapunov exponent, Poincaré map, spectrum map, and bifurcation diagram. Moreover, a new fixed-time convergence theorem is proposed for general chaotic systems based on finite-time control theory, and a fixed-time controller is also put to achieve synchronization of the new chaotic system. Simulation results are presented to show the effectiveness of the theoretical results. The conclusion of the paper is useful for the nonlinear economics and engineering application.
\end{abstract}

\section{Introduction}

After the first chaotic attractor was discovered by Lorenz, people began to search for new chaotic attractors with great enthusiasm. In recent years, for autonomous threedimensional ordinary differential equations, the search for new chaotic attractors has attracted more and more attention. For example, Lorenz system [1], Rossler system [2], Chen and Ueta system [3], Lü and Chen system [4], and Liu et al. system [5]. In a three-dimensional autonomous system, all kinds of chaotic systems with distinct features were put forward, such as chaotic systems with only five terms [6], chaotic systems with linear terms [7], and chaotic systems with only one equilibrium point [8]. In practical engineering applications, we hope that the structure of the chaotic system is as simple as possible based on the consideration implementation; for example, Lü et al. have proposed a unified chaotic system with a single parameter with a simple structure, and it has been well applied in practice $[9,10]$. Therefore, how to construct a simple chaotic system is still a meaningful work in engineering.
Furthermore, research on synchronization and control of the chaos system is particularly topical for decades [11-13]. And, people came to know about the value of achieving control of chaos systems in finite time in some domains of engineering. [14-20]. It is necessary to give the initial conditions of the network in advance, owing to that the establishment time of the finite-time control depends on the initial state of the network to a great extent. However, the initial conditions may be random, so it is not easy to solve practical problems. In order to compensate for this, Polyakov produced the fixed-time stability of the system [21], which ensures that the system is without respect to the initial conditions in a bounded time. In the past few years, a lot of fixed-time stability theories have been raised to study fixedtime stability of the system [22-24]. Inspired by the existing research results, we study fixed-time synchronization of the chaos system. The aim of this paper is to present a new single-parameter chaotic system with a simple structure, and some basic dynamic behavior of the new system is discussed. The synchronization of the new chaotic system is realized by using the new fixed-time controller in this paper. Compared with the existing finite-time synchronization methods of 
chaotic systems, the fixed-time synchronization method proposed in this paper is more general.

This paper is organized as follows: Section 2 gives the new single-parameter chaos system. Section 3 discusses some basic characters of the new chaotic system. Section 4 puts forward the fixed-time control condition for a general chaos system. Section 5 gives an illustrative example. The conclusions are given in Section 6.

\section{The New Single-Parameter Chaos System}

Consider the following system:

$$
\left\{\begin{array}{l}
\dot{x}=y-x, \\
\dot{y}=-x z \\
\dot{z}=x y-a,
\end{array}\right.
$$

which is chaotic for $0.16<a<4.8$ (see Figures $1-4$ for $a=0.5$ ).

\section{Some Basic Dynamic Properties of New Chaotic Systems}

In this section, some basic characteristics of the new chaotic system are analyzed.

\subsection{Equilibria. Let}

$$
\left\{\begin{array}{l}
y-x=0 \\
-x z=0 \\
x y-a=0
\end{array}\right.
$$

When $a>0$, the two equilibrium points of the system can be expressed as $E_{1}=(\sqrt{a}, \sqrt{a}, 0)$ and $E_{2}=(-\sqrt{a},-\sqrt{a}, 0)$.

Let $a=0.5$, and it is easy to obtain the following form of system equilibrium $E_{1}=(\sqrt{2} / 2, \sqrt{2} / 2,0)$ and $E_{2}=(-\sqrt{2} / 2,-\sqrt{2} / 2,0)$.

For $E_{1}=(\sqrt{2} / 2, \sqrt{2} / 2,0)$, system (1) is linearized, and the Jacobian matrix is defined as $J=\left(\begin{array}{ccc}-1 & 1 & 0 \\ -z & 0 & -x \\ y & x & 0\end{array}\right)$.

Let $|\lambda I-J|=0$ to get the eigenvalues of matrix $J^{1}$.

Therefore, when $E_{1}=(\sqrt{2} / 2, \sqrt{2} / 2,0)$, the eigenvalues of matrix $J$ are

$$
\begin{aligned}
& \lambda_{1}=-1.2442, \\
& \lambda_{2}=0.1221+0.8882 i, \\
& \lambda_{2}=0.1221-0.8882 i .
\end{aligned}
$$

Similarly, when $E_{2}=(-\sqrt{2} / 2,-\sqrt{2} / 2,0)$, the eigenvalues of matrix $J$ are

$$
\begin{aligned}
& \lambda_{1}=-1.2442, \\
& \lambda_{2}=0.1221+0.8882 i, \\
& \lambda_{2}=0.1221-0.8882 i .
\end{aligned}
$$

According to equations (3) and (4), it can be determined that both $E_{1}$ and $E_{2}$ are saddle focus nodes.

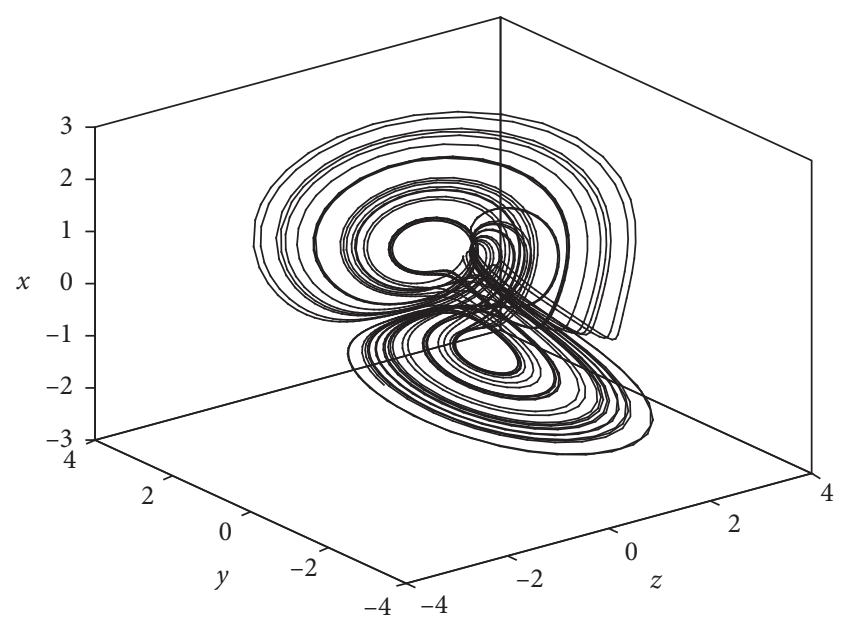

FIgURE 1: A new chaotic attractor.

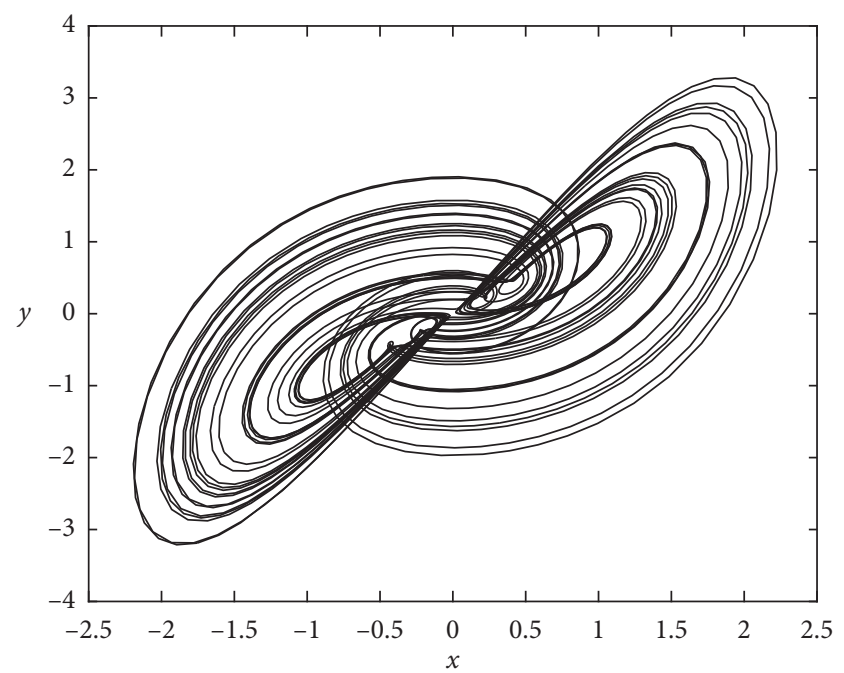

Figure 2: $x-y$ phase plane of the chaotic attractor.

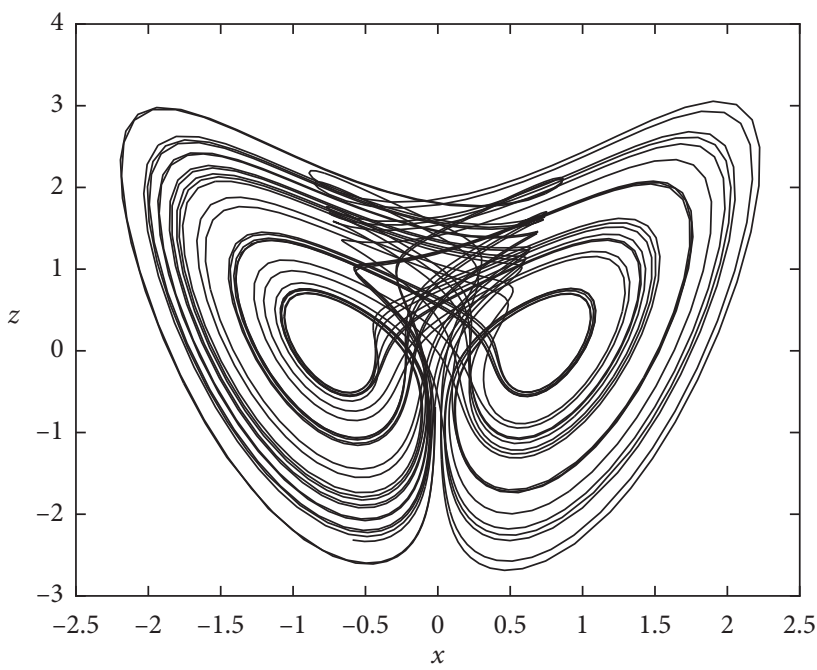

Figure 3: $x-z$ phase plane of the chaotic attractor. 


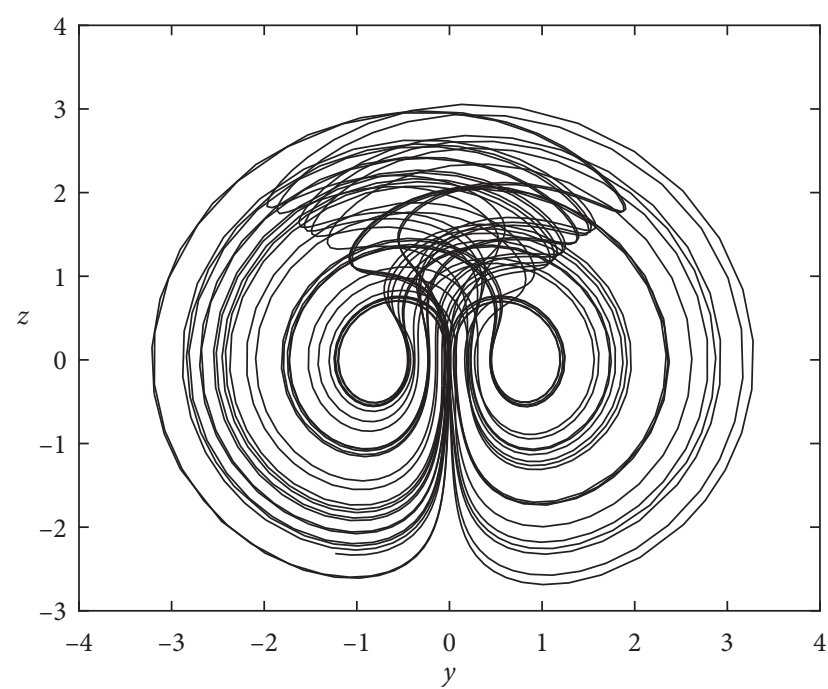

FIgURE 4: $y-z$ phase plane of the chaotic attractor.

3.2. Dissipativity and the Existence of Attractor. For dynamical system (1), we can obtain

$$
\nabla \cdot V=\frac{\partial \dot{x}}{\partial x}+\frac{\partial \dot{y}}{\partial y}+\frac{\partial \dot{z}}{\partial z}=-1
$$

where -1 is a negative value. System (1) is one dissipative system, and an exponential contraction of system (1) is $(\mathrm{d} V / \mathrm{d} t)=e^{-1}$. This means that every volume containing the trajectory of the dynamic system will be reduced to zero as $t \longrightarrow \infty$ at the exponential rate -1 , and the asymptotic motion is located on the attractor of system (1).

3.3. Lyapunov Exponent. It is generally true that a system is chaotic as long as at least one of its Lyapunov exponents is positive. When the initial value is $(1,1,1)$, the Lyapunov exponent of system (1) is calculated to be $L_{1}=0.7125$, $L_{2}=0.0082$, and $L_{3}=-0.4486$, and its dimension is

$$
D_{L}=j+\frac{1}{\left|L_{j+1}\right|} \sum_{i=1}^{j} L_{i}=2+\frac{L_{1}+L_{2}}{\left|L_{3}\right|}=2+\frac{0.7207}{|-0.4486|}=3.6066 .
$$

It implies that system (1) is a dissipative system. Moreover, its Lyapunov dimension is fractional. The fractal character of the attractor means that the system has aperiodic orbits, and the orbits near it are divergent.

3.4. Poincaré Map, Spectrum Map, and Bifurcation Diagram. In Figure 5, the continuous broadband feature is exhibited for the spectrum of system (1). For $z=0, x=0$, and $y=0$, Figures 6(a)-6(c)visualize the Poincaré map in planes, respectively, and several sheets of the attractors are displayed. This can better sum up all the possible behaviors by the bifurcation graph when the parameters on a graph are changing. For $0 \leq a \leq 8$, Figure 7 is a bifurcation diagram of system (1), which shows its complex bifurcation phenomena.

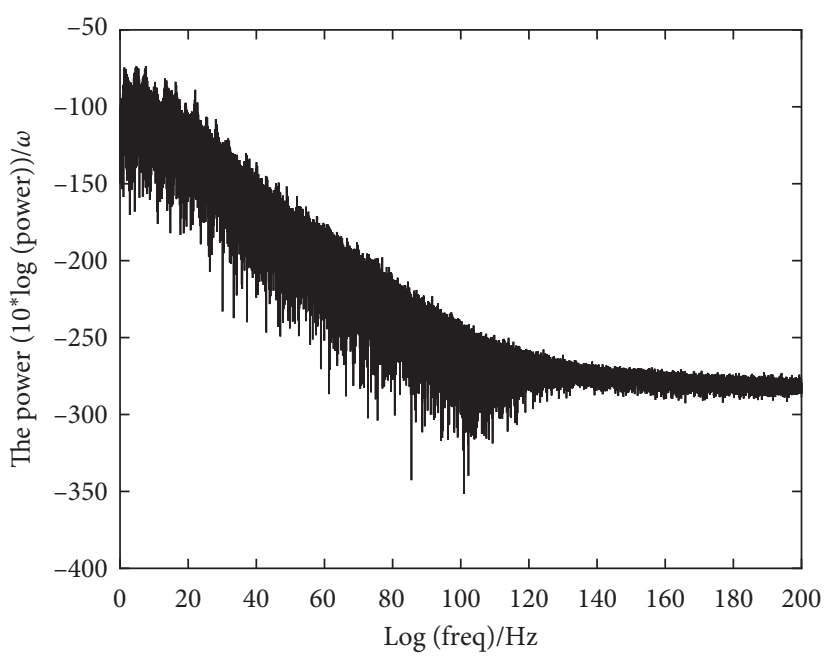

FIgURE 5: An apparently continuous broadband frequency spectrum.

\section{Fixed-Time Synchronization of the General Chaotic System}

Considering the following chaotic system in a general form:

$$
\dot{x}=f(x, t) \text {, }
$$

where $x=\left(x_{1}, \ldots, x_{n}\right)^{T}$ and $f(x): R^{m} \longrightarrow R^{m}$ is a continuous function.

So, the controlled chaos system is given by

$$
\dot{y}=f(y, t)+r(x, y, t),
$$

where $y=\left(y_{1}, \ldots, y_{n}\right)^{T}$ and $r(x, y, t)$ is the controller.

Then, the error system can be

$$
\dot{e}=f(y, t)-f(x, t)+r(x, y, t) .
$$

Assumptions 1. We also assume that $f$ is Lipschitz with respect to its argument, i.e.,

$$
|f(t, y)-f(t, x)| \leq \eta e, \quad \eta \in R,
$$

where

$e=\left(e_{1}, e_{2}, \ldots, e_{n}\right)^{T}=\left(y_{1}-x_{1}, y_{2}-x_{2}, \ldots, y_{n}-x_{n}\right)^{T}$.

Lemma 1 (see [21]). For $\delta_{i} \geq 0, i=1, \ldots, n, 0<\varsigma \leq 1$, and $\theta>1$, then

$$
\begin{aligned}
& \sum_{i=1}^{n} \delta_{i}^{\varsigma} \geq\left(\sum_{i=1}^{n} \delta_{i}\right)^{\varsigma}, \\
& \sum_{i=1}^{n} \delta_{i}^{\theta} \geq n^{1-\theta}\left(\sum_{i=1}^{n} \delta_{i}\right)^{\theta} .
\end{aligned}
$$

Lemma 2 (see [21]). Assume that a continuous and a positive-definite function $v(t)$ satisfies

$$
\dot{v}(t) \leq-\mu v^{p}(t), \quad t \geq t_{0}, v\left(t_{0}\right) \geq 0,
$$




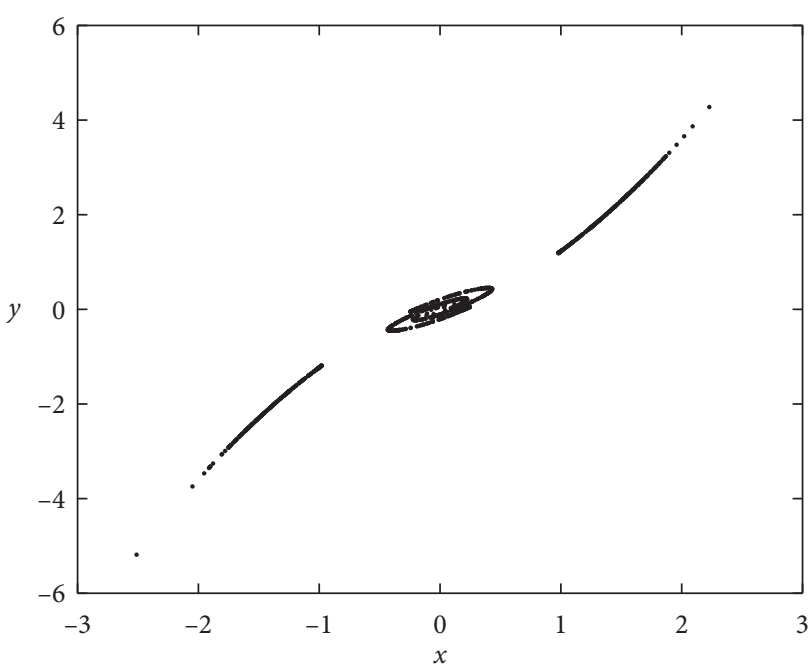

(a)

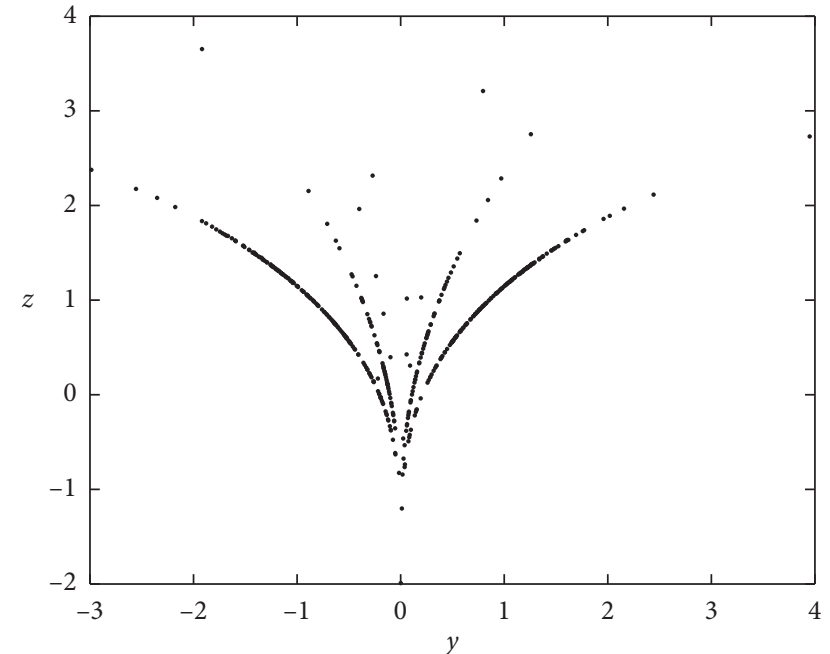

(b)

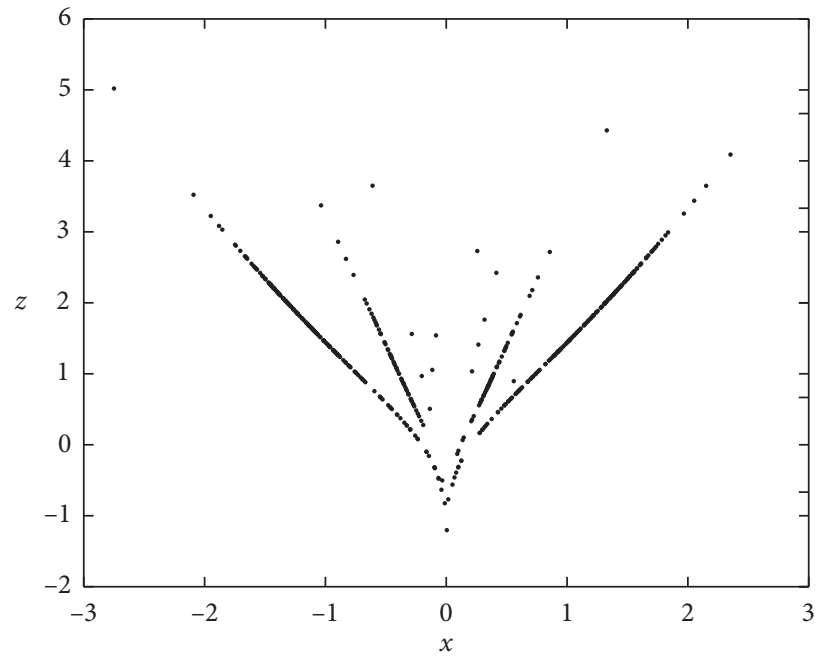

(c)

Figure 6: Poincaré maps for (a) $z=0$, (b) $x=0$, and (c) $y=0$.

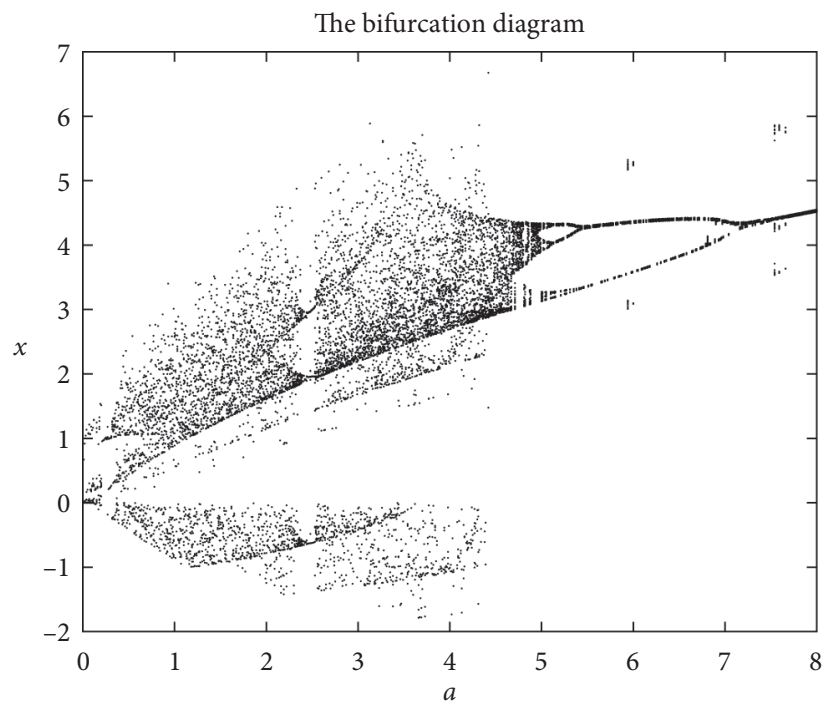

FIgURE 7: Bifurcation diagram of system (1) for $0 \leq a \leq 8$. 
where $\mu>0$ and $0<p<1$ are all constants. Then, for any given $t_{0}, v(t)$ satisfies

$$
\begin{aligned}
v^{1-p}(t) & \leq v^{1-p}\left(t_{0}\right)-\mu(1-p)\left(t-t_{0}\right), \quad t_{0} \leq t \leq t_{1}, \\
v(t) & =0, \quad t \geq T_{1},
\end{aligned}
$$

with $T_{1}$ given by

$$
T_{1}=t_{0}+\frac{v^{1-p}\left(t_{0}\right)}{\mu(1-p)}
$$

In the following, the new fixed-time controller based on Lemma 2 is proposed.

Theorem 1. Assuming that hypothesis 1 holds, two chaotic systems (7) and (8) can be synchronized by the following fixedtime controllers:

$$
r(t)= \begin{cases}-\frac{k_{1}}{\|e\|^{2}} e-k_{2} e^{1+k_{3} \operatorname{sign}(\|e\|-1)}, & \|e\| \neq 0, \\ 0, & \|e\|=0,\end{cases}
$$

and the time is bounded as

$$
T \leq \frac{1}{k_{1}}+\frac{1}{k_{2} n^{\left(-k_{3} / 2\right)} 2^{\left(2+k_{3}\right) / 2}-2 \eta} \frac{2}{k_{3}},
$$

where $k_{1}>2 \eta, k_{2}>2 \eta$, and $0<k_{3}<1$.

Proof. Let

$$
v(t)=\frac{1}{2} e^{T}(t) e(t)
$$

Then,

$$
\begin{aligned}
\dot{v}(t) & =e^{T}(t) \dot{e}(t)=e^{T}(t)(f(v(t))-f(u(t))+r(t)) \\
& \leq \eta e^{T}(t) e(t)-k_{1}-k_{2} e^{T}(t) e^{1+k_{3} s i g n(\|e\|-1)} \\
& = \begin{cases}\eta e^{T}(t) e(t)-k_{1}-k_{2} e^{T}(t) e^{1+k_{3}}, & \|e\|>1, \\
\eta e^{T}(t) e(t)-k_{1}-k_{2} e^{T}(t) e^{1-k_{3}}, & \|e\|<1 .\end{cases}
\end{aligned}
$$

Obviously,

$$
\begin{aligned}
& -k_{2} e^{T} e^{1+k_{3}} \leq-k_{2} n^{\left(-k_{3} / 2\right)}\left(e^{T} e\right)^{\left(2+k_{3}\right) / 2}= \\
& \quad-k_{2} n^{\left(-k_{3} / 2\right)} 2^{\left(2+k_{3}\right) / 2} v^{\left(2+k_{3}\right) / 2}, \\
& -k_{2} e^{T} e^{1-k_{3}} \leq-k_{2}\left(e^{T} e\right)^{\left(2-k_{3}\right) / 2}=-k_{2} 2^{\left(2-k_{3}\right) / 2} v^{\left(2-k_{3}\right) / 2} .
\end{aligned}
$$

So,

$$
\dot{v} \leq \begin{cases}2 \eta v-k_{1}-k_{2} n^{\left(-k_{3} / 2\right)} 2^{\left(2+k_{3}\right) / 2} v^{\left(2+k_{3}\right) / 2}, & \|e\|>1, \\ 2 \eta v-k_{1}-k_{2} 2^{\left(2-k_{3}\right) / 2} v^{\left(2-k_{3}\right) / 2}, & \|e\|<1 .\end{cases}
$$

Let $\Phi(v)=v^{2}$, then

$$
\dot{\omega}(v)=2 v \dot{v} \leq \begin{cases}2 v\left(2 \eta v-k_{1}-k_{2} n^{\left(-k_{3} / 2\right)} 2^{\left(2+k_{3}\right) / 2} v^{\left(2+k_{3}\right) / 2}\right), & \|e\|>1, \\ 2 v\left(2 \eta v-k_{1}-k_{2} 2^{\left(2-k_{3}\right) / 2} v^{\left(2-k_{3}\right) / 2}\right), & \|e\|<1 .\end{cases}
$$

When $k_{1}>2 \eta$ and $k_{2}>2 \eta$, from Lemma 2 , we have

$$
\begin{aligned}
2 \eta v & -k_{1}-k_{2} n^{\left(-k_{3} / 2\right)} 2^{\left(2+k_{3}\right) / 2} v^{\left(2+k_{3}\right) / 2} \\
& \leq-\left[\kappa_{1}^{2 /\left(2+k_{3}\right)}-2^{2 /\left(2+k_{3}\right)} \eta^{2 /\left(2+k_{3}\right)} v^{2 /\left(2+k_{3}\right)}\right. \\
& \left.+2 k_{2}^{2 /\left(2+k_{3}\right)} n^{-k_{3} /\left(2+k_{3}\right)} v\right]^{\left(2+k_{3}\right) / 2},
\end{aligned}
$$

$$
\begin{aligned}
2 \eta v & -k_{1}-k_{2} 2^{\left(2-k_{3}\right) / 2} v^{\left(2-k_{3}\right) / 2} \\
& \leq-\left[k_{1}^{2 /\left(2-k_{3}\right)}-2^{2 /\left(2-k_{3}\right)} \eta^{2 /\left(2-k_{3}\right)} v^{2 /\left(2-k_{3}\right)}\right. \\
& \left.+2 k_{2}^{2 /\left(2-k_{3}\right)} v\right]^{\left(2-k_{3}\right) / 2} .
\end{aligned}
$$

By (23)-(24),

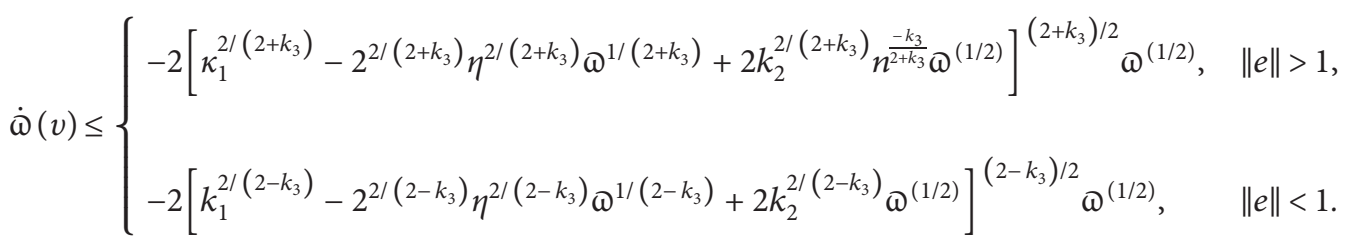

If

$$
k_{1}>2 \eta, k_{2}>2 \eta,
$$
$\dot{\omega}(v) \leq\left\{\begin{array}{ll}-2 k_{1} \varpi^{(1 / 2)}, & \|e\|>1, \\ -2^{\left(4-k_{3}\right) / 2} k_{2} \varpi^{\left(4-k_{3}\right) / 4}, & \|e\|<1,\end{array}\right.$ it is known from Lemma 2 that the zero solution of system (9) is fixed-time stable.

From (21),

$$
\frac{\mathrm{d} v}{\mathrm{~d} t} \leq \begin{cases}2 \eta v-k_{1}-k_{2} n^{\left(-k_{3} / 2\right)} 2^{\left(2+k_{3}\right) / 2} v^{\left(2+k_{3}\right) / 2}, & \|e\|>1, \\ 2 \eta v-k_{1}-k_{2} 2^{\left(2-k_{3}\right) / 2} v^{\left(2-k_{3}\right) / 2}, & \|e\|<1 .\end{cases}
$$

So, 

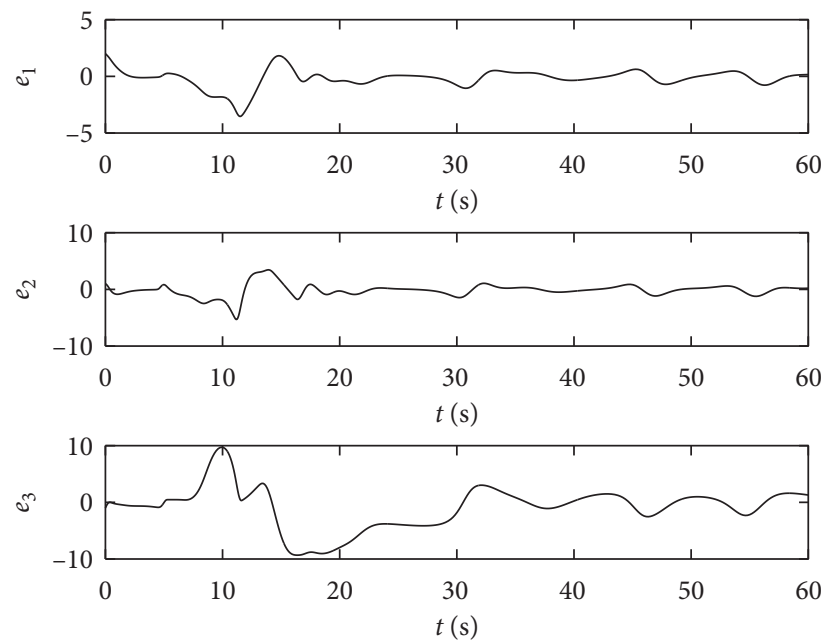

FIGURE 8: Error evolution without the controller.

$\mathrm{d} t \leq \begin{cases}\frac{\mathrm{d} v}{2 \eta v-k_{1}-k_{2} n^{\left(-k_{3} / 2\right)} 2^{\left(2+k_{3}\right) / 2} v^{\left(2+k_{3}\right) / 2}}, & \|e\|>1, \\ \frac{\mathrm{d} v}{2 \eta v-k_{1}-k_{2} 2^{\left(2-k_{3}\right) / 2} v^{\left(2-k_{3}\right) / 2},} & \|e\|<1 .\end{cases}$
When $T, v(t) \longrightarrow 0$, the settling time $T$ can be estimated as

$$
\begin{aligned}
T \leq & \int_{0}^{1} \frac{\mathrm{d} v}{k_{1}-2 \eta v+k_{2} 2^{\left(2-k_{3}\right) / 2} v^{\left(2-k_{3}\right) / 2}}+\int_{1}^{v(0)} \frac{\mathrm{d} v}{k_{1}-2 \eta v+k_{2} n^{\left(-k_{3} / 2\right)} 2^{\left(2+k_{3}\right) / 2} v^{\left(2+k_{3}\right) / 2}} \leq \int_{0}^{1} \frac{\mathrm{d} v}{k_{1}} \\
& +\int_{1}^{v(0)} \frac{\mathrm{d} v}{\left(k_{2} n^{\left(-k_{3} / 2\right)} 2^{\left(2+k_{3}\right) / 2}-2 \eta\right) v^{\left(2+k_{3}\right) / 2}} .
\end{aligned}
$$

When $v^{\left(2+k_{3}\right) / 2}>1, \int_{1}^{v(0)}\left(\mathrm{d} v / v^{\left(2+k_{3}\right) / 2}\right)<2 / k_{3}$, so

$$
T \leq \frac{1}{k_{1}}+\frac{1}{k_{2} n^{\left(-k_{3} / 2\right)} 2^{\left(2+k_{3}\right) / 2}-2 \eta} \frac{2}{k_{3}} \text {. }
$$

The proof is completed.

Remark 1. In [12-14], different finite-time controllers are designed for different chaotic systems. The fixed-time controllers designed in this paper are more general and can be used in other different chaotic systems.

Remark 2. For the complex dynamic network satisfying Assumption 1, the method of Theorem 1 can be used to control the complex network.

\section{Simulation and Results}

We assume that the new chaotic system is the drive system, that is,

$$
\left\{\begin{array}{l}
\dot{u}_{1}=u_{2}-u_{1}, \\
\dot{u}_{2}=-u_{1} u_{3}, \\
\dot{u}_{3}=u_{1} u_{2} .
\end{array}\right.
$$

And, the response system is

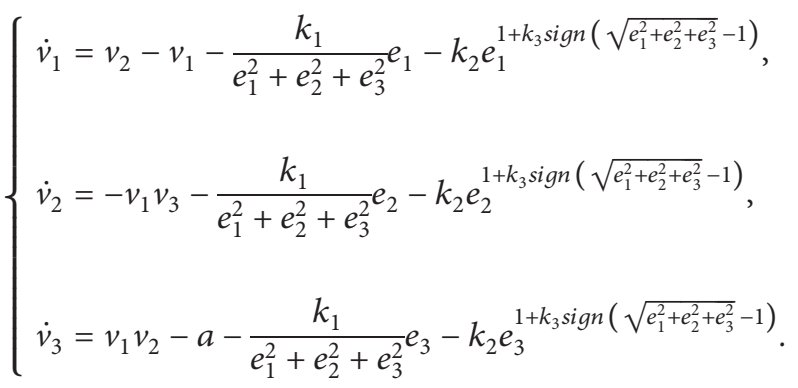

The initial conditions of the master and slave systems are $(1,2,1)$ and $(2,1,3)$, respectively. Through simulation calculation, $\eta \approx 58.2516$, and let $k_{1}=117, k_{2}=118$, and $k_{3}=0.5$. Synchronization of two chaotic systems cannot be achieved without a controller as shown in Figure 8. The 

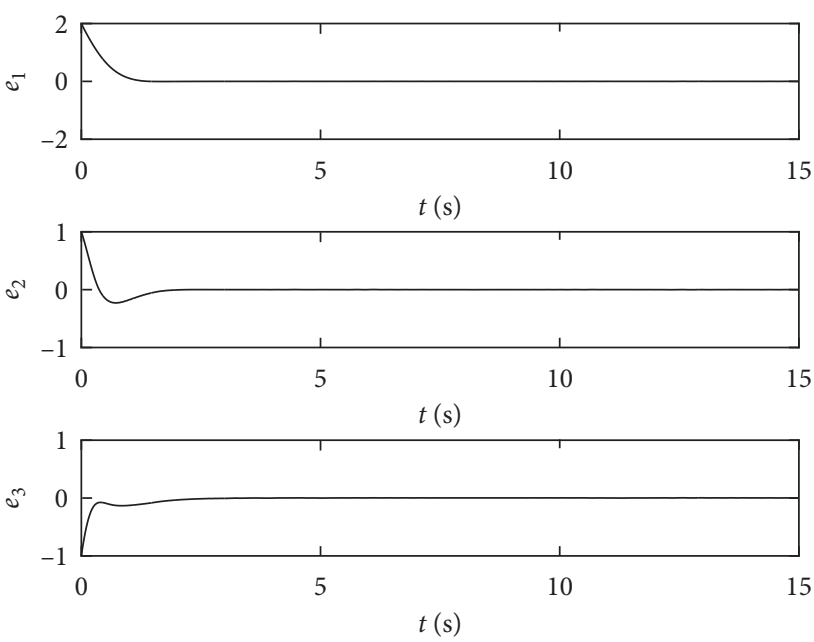

FIgURE 9: Synchronization errors.

synchronization evolution of two chaos systems is shown in Figure 9. Numerical simulations illustrate the validity of Theorem 1.

\section{Conclusion}

The paper has presented a new chaotic system with a single parameter and discussed some basic dynamic behavior of the new system. Base on finite-time control theory, the fixedtime synchronization criterion of the new chaotic system has also been obtained. Finally, the result of numerical simulation proves that they are feasible. In the future, we will discuss the fixed-time synchronization of general complex networks.

\section{Data Availability}

No data were used to support this study.

\section{Conflicts of Interest}

The authors declare that there are no conflicts of interest regarding the publication of this paper.

\section{Acknowledgments}

This work was supported by the National Natural Science Foundation of China (61673221); the Natural Science Foundation of Jiangsu Province (BK20181418); the Natural Science Foundation of Jiangsu Higher Education Institutions (19KJB120007); the Six Talent Peaks Project in Jiangsu Province (DZXX-019).

\section{References}

[1] E. N. Lorenz, "Deterministic nonperiodic flow," Journal of the Atmospheric Sciences, vol. 20, no. 2, pp. 130-141, 1963.

[2] O. E. Rössler, "An equation for continuous chaos," Physics Letters A, vol. 57, no. 4, pp. 397-398, 1976.
[3] G. Chen and T. Ueta, "Yet another chaotic attractor," International Journal of Bifurcation and Chaos, vol. 9, no. 7, pp. 1465-1466, 1999.

[4] J. Lü and G. Chen, "A new chaotic attractor coined," International Journal of Bifurcation and Chaos, vol. 12, no. 3, pp. 659-661, 2002.

[5] C. Liu, T. Liu, L. Liu, and K. Liu, "A new chaotic attractor," Chaos, Soliton and Fractals, vol. 22, no. 5, pp. 1031-1038, 2004.

[6] B. Munmuangsaen and B. Srisuchinwong, "A new five-term simple chaotic attractor," Physics Letters A, vol. 373, no. 44, pp. 4038-4043, 2009.

[7] Y. Xu and Y. Wang, "A new chaotic system without linear term and its impulsive synchronization," Optik, vol. 125, no. 11, pp. 2526-2530, 2014.

[8] Z. Wei, "Dynamical behaviors of a chaotic system with no equilibria," Physics Letters A, vol. 376, no. 2, pp. 102-108, 2011.

[9] J. Lü, G. Chen, D. Cheng, and S. Celikovsky, "Bridge the gap between the Lorenz system and the Chen system," International Journal of Bifurcation and Chaos, vol. 12, no. 12, pp. 2917-2926, 2002.

[10] Q. Lai and S. Chen, "Generating multiple chaotic attractors from Sprott B system," International Journal of Bifurcation and Chaos, vol. 26, no. 11, Article ID 1650177, 2016.

[11] Y. Xu, C. Xie, and Q. Xia, "A kind of binary scaling function projective lag synchronization of chaotic systems with stochastic perturbation," Nonlinear Dynamics, vol. 77, no. 3, pp. 891-897, 2014.

[12] V. K. Yadav, V. K. Shukla, and S. Das, "Difference synchronization among three chaotic systems with exponential term and its chaos control," Chaos, Solitons and Fractals, vol. 124, pp. 36-51, 2019.

[13] Y. Zhang and T. Jiang, "Finite-time boundedness and chaos-like dynamics of a class of Markovian jump linear systems," Journal of the Franklin Institute, vol. 357, no. 4, pp. 2083-2098, 2020.

[14] R. Sakthivel, S. Santra, B. Kaviarasan, and J. H. Park, "Finitetime sampled-data control of permanent magnet synchronous motor systems," Nonlinear Dynamics, vol. 86, no. 3, pp. 2081-2092, 2016.

[15] S. Mobayen and J. Ma, "Robust finite-time composite nonlinear feedback control for synchronization of uncertain chaotic systems with nonlinearity and time-delay," Chaos, Solitons and Fractals, vol. 114, pp. 46-54, 2018.

[16] Y. Li, X. Wu, J.-A. Lu, and J. Lü, "Synchronizability of duplex networks," IEEE Transactions on Circuits and Systems II: Express Briefs, vol. 63, no. 2, pp. 206-210, 2016.

[17] J. Ni, L. Liu, C. Liu, X. Hu, and S. Li, "Fast fixed-time nonsingular terminal sliding mode control and its application to chaos suppression in power system," IEEE Transactions on Circuits and Systems II: Express Briefs, vol. 64, no. 2, pp. 151-155, 2017.

[18] I. Ahmad, M. Shafiq, A. B. Saaban, A. B. Ibrahim, and M. Shahzad, "Robust finite-time global synchronization of chaotic systems with different orders," Optik, vol. 127, no. 19, pp. 8172-8185, 2016.

[19] Y. Xu, W. Zhou, J. Fang, C. Xie, and D. Tong, "Finite-time synchronization of the complex dynamical network with nonderivative and derivative coupling," Neurocomputing, vol. 173, pp. 1356-1361, 2016.

[20] Y. Xu, Z. Ke, C. Xie, and W. Zhou, "Dynamic evolution analysis of stock price fluctuation and iIts control," Complexity, vol. 2018, Article ID 5728090, 9 pages, 2018.

[21] A. Polyakov, "Nonlinear feedback design for fixed-time stabilization of linear control systems," IEEE Transactions on Automatic Control, vol. 57, no. 8, pp. 2106-2110, 2012. 
[22] Y. Xu, X. Wu, N. Li, L. Liu, C. Xie, and C. Li, "Fixed-time synchronization of complex networks with a simpler nonchattering controller," IEEE Transactions on Circuits and Systems II: Express Briefs, vol. 67, no. 4, pp. 700-704, 2020.

[23] J. Cao and R. Li, "Fixed-time synchronization of delayed memristor-based recurrent neural networks," Science China Information Sciences, vol. 60, no. 3, Article ID 032201, 2017.

[24] Y. Xu, X. Wu, B. Mao, and C. Xie, "A unified finite-/fixed-time synchronization approach to multi-layer networks," IEEE Transactions on Circuits and Systems II: Express Briefs, p. 1, 2020, In press. 